\title{
Binary corrected X-ray light curve of Cygnus X-3: Implications for the timing properties of the compact binary system
}

\author{
M. Choudhury ${ }^{1}$, A. R. Rao ${ }^{1}$, S. V. Vadawale ${ }^{1, \star}$, A. K. Jain ${ }^{2}$, and N. S. Singh ${ }^{3}$ \\ 1 Tata Institute of Fundamental Research, Homi Bhabha Road, Mumbai-400005, India \\ 2 ISRO Satellite Centre, Bangalore-560017, India \\ 3 Dept. of Physics, Manipur University, Canchipur, Imphal, Manipur-795003, India
}

Received 5 May 2003 / Accepted 9 March 2004

\begin{abstract}
We study the binary corrected X-ray light curve of Cygnus X-3 using the RXTE-ASM (All Sky Monitor) data and RXTE-PCA data. We find that the X-ray template derived from the EXOSAT observations adequately explains the binary variations. Using the recently determined quadratic binary ephemeris we correct the RXTE-PCA lightcurve and obtain the power spectrum which shows distinct features of shifting towards the low frequency regime vis-a-vis the Galactic X-ray binaries. The power spectrum doesn't vary from the hard to the soft state. We also obtain the binary corrected RXTE-ASM monitoring lightcurve and examine the previously obtained correlation between soft X-ray (2-12 keV from RXTE ASM), hard X-ray (20-100 keV from CGRO-BATSE) and radio (2.2 GHz from GBI) after the binary correction. We find that the correlation time scale is less than a day.
\end{abstract}

Key words. accretion, accretion disks - stars: binaries: close - stars: individual: Cygnus X-3 - radio continuum: stars X-rays: binaries

\section{Introduction}

Cygnus X-3, first discovered as a bright X-ray source (Giacconi et al. 1967), is an extensively studied Galactic binary system, being one of the brightest source in the X-ray, infrared and radio regions of the electromagnetic spectrum (see Bonnet-Bidaud \& Chardin 1988 and the references therein). It shows a strong $4.8 \mathrm{~h}$ modulation in the X-ray (Parsignault et al. 1972) and the infra-red emission which is attributed to the binary orbital motion (Becklin et al. 1973). The measurement of the radial velocity in the infra-red band and its interpretation as due to binary Doppler shift has led to the derivation of a large mass function for the source (Schmutz et al. 1996), but this simplistic explanation of the He II line shift is strongly disputed by the relative phasing of the infra-red and the X-ray binary modulation (van Kerkwijk 1993). If the spectral features observed in the infrared are ascribed to a mass losing Wolf-Rayet like companion, a large mass loss, large mass for the companion and $\sim 20 M_{\odot}$ mass for the primary (suggesting Cygnus X-3 as a black hole source) can be derived (van Kerkwijk et al. 1992). Mitra (1996), however, has pointed out that these derived parameters are incompatible with observed X-ray properties of the source. Interestingly, very recently Stark \& Saia (2003)

Send offprint requests to: M. Choudhury,

e-mail: manojulu@tifr.res.in

* Present address: Harvard Smithsonian Center for Astrophysics, 60 Garden Street, Cambridge, MA 02138, USA. have suggested an upper limit to the mass of the compact object of 3.6 $M_{\odot}$. They measured the Doppler shift of the He $\alpha$ like line of Fe XXV (Paerels et al. 2000; Kitamoto et al. 1994) and ascribing it to originate from very near the surface of the compact object they provide a conservative estimate of the stellar masses and the separation of the binary system.

The $4.8 \mathrm{~h} \mathrm{X-ray} \mathrm{light} \mathrm{curve} \mathrm{has} \mathrm{shown} \mathrm{a} \mathrm{remarkable} \mathrm{sta-}$ bility in terms of its shape (Ghosh et al. 1981) and the period has shown a gradual increase at a rate of $\sim 10^{-9} \mathrm{~s} \mathrm{~s}^{-1}$. Recently, Singh et al. (2002) have extended the span of the observation and have concluded that the period change is consistent with a constant rate of $5.76 \pm 0.24 \times 10^{-10}$. Remarkably, the binary modulation was also found to be consistent with the template derived from the EXOSAT data (van der Klis \& Bonnet-Bidaud 1989).

The radio emission shows flaring activity (Gregory et al. 1972; Watanabe et al. 1994; McCollough et al. 1999) during which it exhibits radio-jets analogous to those of powerful radio galaxies and quasars (Waltman et al. 1995; Fender et al.1997). There have been several attempts to connect the radio, X-ray, and infrared variations in the source (Watanabe et al. 1994; Fender 1997). Recently Choudhury et al. (2002), have reported a strong and significant correlation between the radio and X-ray emission in the low-hard state of Cygnus X-3. They concluded that the radio emission is related to the shape of the X-ray spectrum which pivots at around $12 \mathrm{keV}$ with the $\mathrm{X}$-ray photons softer than the pivot energy highly correlated to 


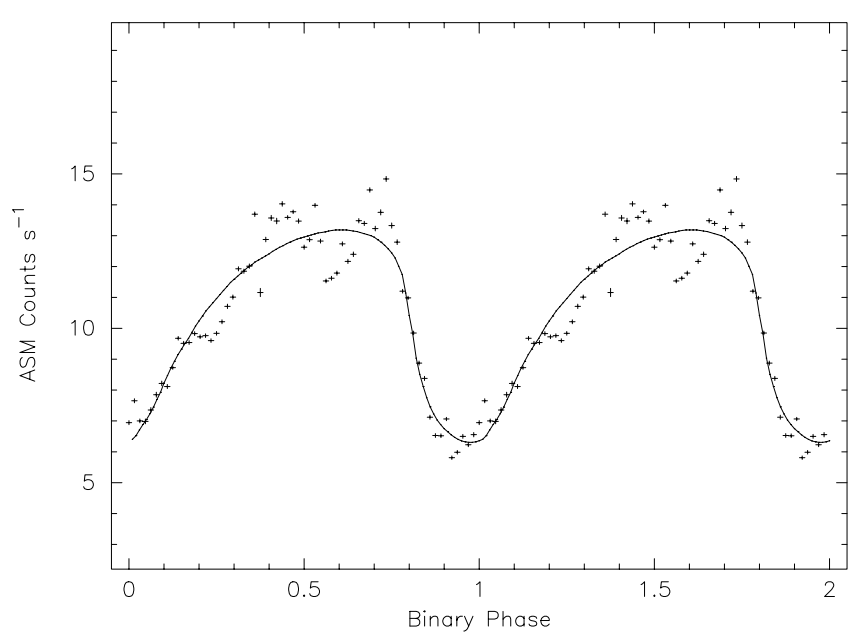

Fig. 1. The X-ray light curve of Cygnus X-3 obtained from RXTE-ASM for the period of MJD 50410 to 51400 folded at the quadratic ephemeris given by Singh et al. (2002). The template data obtained from EXOSAT (van der Klis \& Bonnet-Bidaud 1989) is also shown, with appropriate scaling for the count rates.

the radio emission. This signifies a definite connection between the inflow of matter via accretion disk emission and the outflow via jet emission (Choudhury et al. 2003).

In this paper we develop a method to correct for the variation in the X-ray emission due to the binary modulation in this source and thereafter study the X-ray timing characteristics. We also investigate the above mentioned radio: X-ray correlation of the source (Choudhury et al. 2002) after correcting for the binary modulation in the X-ray and conclude that the correlation time scale is shorter than one day.

\section{Binary correction}

The evolution of the $4.8 \mathrm{~h}$ binary period of Cygnus X-3 has been studied extensively with the time derivative $(\dot{P})$ measured to be $\sim 10^{-9}$ (van der Klis \& Bonnet-Bidaud 1981, 1989; Kitamoto et al. 1987). Recently, Singh et al. (2002) have extended the data base of the binary period measurements using the Indian X-ray Astronomy Experiment (IXAE) and archival data from ROSAT, ASCA, BeppoSAX and RXTE. They found that the binary template obtained from the EXOSAT data adequately explains the recent observations and have derived a value of the period derivative of $5.76 \pm 0.24 \times$ $10^{-10}$. Inclusion of second derivative marginally improved the fit, essentially giving an upper limit to the second derivative $\left(\ddot{P}=-1.3 \pm 1.6 \times 10^{-11} \mathrm{yr}^{-1}\right)$.

We have folded the RXTE ASM data using the quadratic ephemeris of Singh et al. (2002) from MJD 50410 to MJD $51400\left(P=0.19968443 \mathrm{~d} ; \dot{P}=5.76 \times 10^{-10}\right.$; time of zero phase $\left.T_{0}=2440949.892 \mathrm{JD}\right)$. The time span chosen for the folding corresponds to the data used by Choudhury et al. (2003) for the detailed X-ray, radio and hard X-ray correlation analysis. The folded light curve is shown in Fig. 1 (the errors in the data points are smaller than the symbol size). The template data from van der Klis \& Bonnet-Bidaud (1989) is also shown in Fig. 1, after appropriate scaling. The relative phase of the data and template are not arbitrarily shifted for a proper match: the folded light curve (with the above ephemeris) is overlaid with the vertically shifted and scaled template data. The folded count rate correlates with the template value with a correlation coefficient of 0.95 (for 64 data points). It can be seen from the figure that there are non-statistical variations due to the source over and above the binary variation. The ASM count rate, $C$, can be expressed as

$C=10.50 \pm 0.59+3.31 \pm 0.35 V$

where $V$ is the template value for a given phase as given in van der Klis \& Bonnet-Bidaud (1989). The errors are nominal $1 \sigma$ errors obtained by assuming that the fluctuations in each phase bin is random in nature.

During this period, Cygnus X-3 shows several X-ray flares and also transition between low-hard to high-soft states (see Fig. 1 of Choudhury et al. 2002). Considering the large variation of the X-ray flux during the period covered, the folded light curve agrees with the template quite well. We have examined the fluctuations in the source counts over and above the binary variations with respect to binary phase as well as the observation duration. Though there is some evidence for large flare like variations during the phase 0.2 to 0.5 , the rest of the fluctuations occur throughout without any association with binary phase or time of observations. Note also that we have not done any phase fitting for this analysis. Hence we conclude that the error in the phase of light curve minimum is negligible compared to the overall source fluctuations.

Using this information, we can correct each observation for the binary modulation, as follows. The quadratic ephemeris to get the zero phase for the $n t h$ cycle of the period is given as (Singh et al. 2002):

$T_{n}=T_{0}+P_{0} n+c n^{2}$

where $c=\frac{1}{2} P_{0} \dot{P}$. This equation can be inverted to give the binary phase at any time $T$ as,

$n=\frac{\left(T-T_{0}\right)}{P_{0}}-\frac{\left(T-T_{0}\right)^{2} c}{P_{0}^{3}}$.

For the RXTE ASM data, we have calculated the phase using Eq. (3) and from the corresponding template value, corrected for the linear term in Eq. (1). The binary corrected ASM data is again folded and the folded light curve is shown in Fig. 2. It can be seen from the figure that for the long term monitoring data a quadratic ephemeris can explain the modulation quite well and this ephemeris may be used to correct the data for the binary variation. However, we caution, that despite the stable profile with a monotonic change in its period for several years, Cygnus X-3 may show jitters in the individual binary phase measurements (see Singh et al. 2002). This implies that either (a) there is an inherent jitter in the minimum of the binary phase or b) it has a stable profile and short term variability is superimposed on it. We have made the binary correction for the subsequent analysis assuming that Cygnus X-3 has a stable profile. The implication of the possible random phase jitter is examined at appropriate places, if required. 


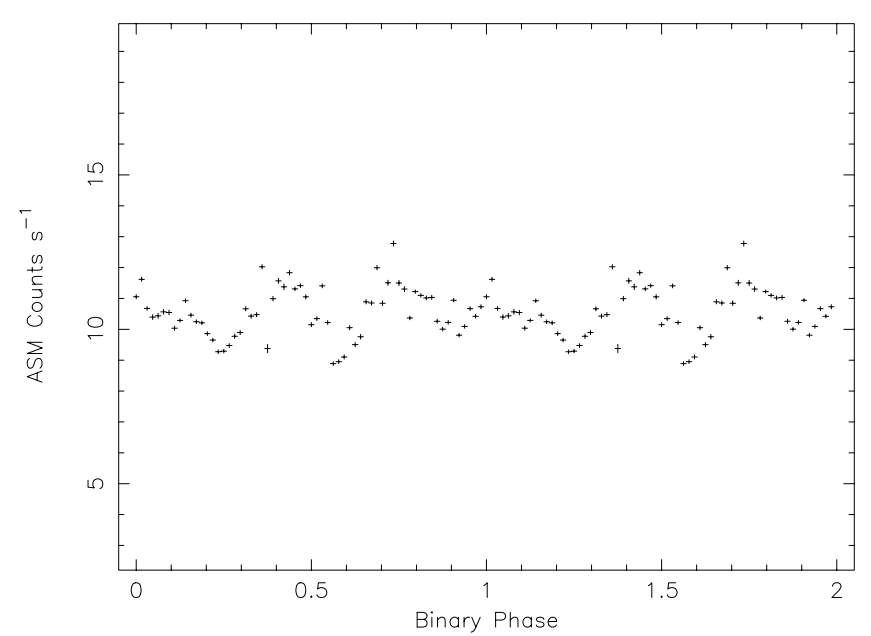

Fig. 2. The X-ray light curve of Cygnus X-3, obtained from RXTE-ASM monitoring data, folded at a binary period of $4.8 \mathrm{~h}$ after the correction for the quadratic ephemeris given by Singh et al. (2002).

We apply the binary correction to the individual RXTE-PCA observations and in Fig. 3 we show both the uncorrected (top panel) and corrected (bottom panel) lightcurve of the single longest observation (Obs. Id. 10126-01-01-020 and 10126-01-01-02) of the source by RXTE. The observation covers about two and a half binary cycles, punctuated by the necessary breaks due to Earth occultation and various other data dropouts. The lightcurve is obtained from single bit data $(2-6.5 \mathrm{keV})$ with all the five PCUs on. The binary template (Fig. 1) is asymmetric with an unusually broad peak lasting in the $\sim 0.4-0.75$ phase of the binary period with a gradual rise before and a steep fall after the peak. It is evident that the correction for binary modulation is very good for the rising and falling phase of the binary ephemeris, highlighting the small variations which were otherwise smothered by the binary modulation. Again we emphasize here that we have not done any fitting for deriving the zero phase, but have used the quadratic ephemeris to derive it. During the peak the lightcurve shows fluctuations not correctable by the smooth peak of the template. This, generally random, fluctuation is an inherent feature of the source present in all the observations, past and present (see van der Klis \& Bonnet-Bidaud 1982, 1989). We employ this binary correction for the timing analysis presented in the following sections.

\section{Temporal characteristics of pointed RXTE observations}

\subsection{Power Density Spectrum}

The X-ray variation of Cygnus X-3 is dominated by the binary modulation and a detailed study of its variability characteristics as a function of frequency and comparison with other black hole candidate sources has not been attempted so far. van der Klis et al. (1985) report the detection of very low frequency regular oscillations during the rising phase of the binary and Rao et al. (1991) report regular oscillations in hard X-rays. Here we present the Power Density Spectrum (PDS) for the

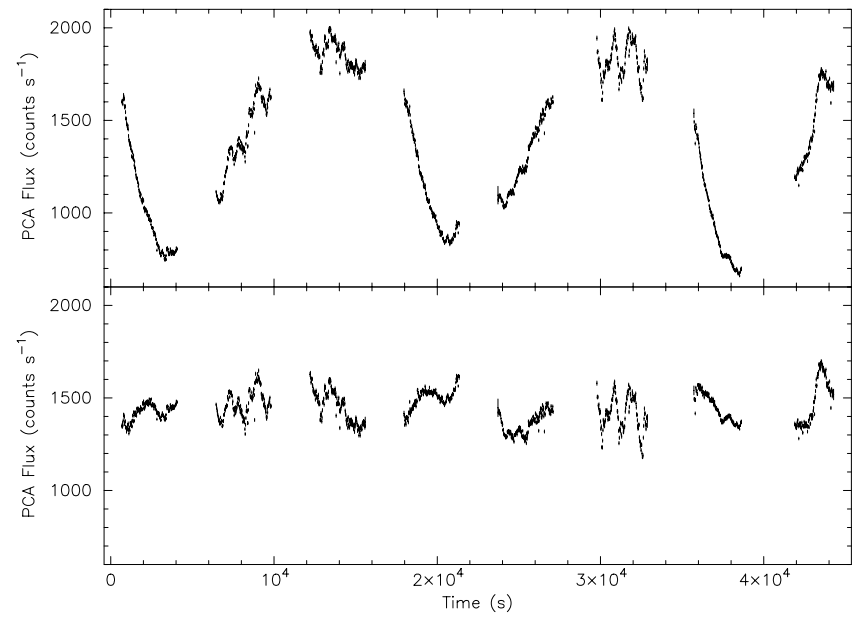

Fig. 3. The X-ray light curve of Cygnus $X-3$ obtained from RXTE-PCA pointed observations (top panel), shown along with light curve (bottom panel) corrected for the binary variation using the quadratic ephemeris given by Singh et al. (2002).

hard states of Cygnus X-3 with emphasis on the duration of the multi wavelength monitoring of Choudhury et al. (2002), after correcting for the binary variability.

To demonstrate the effect of binary correction on the PDS of this source, we again consider the longest continuous stretch of pointed observation of this source shown in Fig. 3 (Obs. Id. 10126-01-01-020 and 10126-01-01-02, MJD 50322). Figure 4 shows the PDS after the binary correction. To explicitly demonstrate that the shape of residual variability is independent of binary phase, we show in Fig. 4 the PDS's separately for the falling part of the light curve (phase 0.75-1.0 top panel), rising part of the light curve (phase 0-0.4 - middle panel), and the peak of the light curve (phase $0.4-0.75$ - bottom panel). Apart from the larger variability due to flares in the rising part (manifest as increased power below $10^{-3} \mathrm{~Hz}$ ), the shape of the PDS are similar for all the three cases. The powerlaw index in the frequency range $10^{-3} \mathrm{~Hz}-0.1 \mathrm{~Hz}$ is consistent with -1.5 and the total rms power is a few per cent. One of the remarkable feature of the light curve is the negligible power above $0.1 \mathrm{~Hz}$, and this feature is observable in the PDS obtained from all the lightcurves, spanning both hard and soft states (Fig. 5). In fact, as shown in Fig. 5, the PDS show the same characteristic featureless powerlaw behaviour in all the states.

\subsection{PDS at low frequencies}

Reig et al. (2002) have examined the aperiodic variability of two micro-quasars Cyg X-1 and GRS 1915+105 at very low frequencies using the RXTE-ASM dwell data. At frequencies below $10^{-5} \mathrm{~Hz}$ it was found that the PDS is consistent with an index of $\sim-1$ and the rms power below $10^{-5} \mathrm{~Hz}$ is $21-27 \%$, for these two sources. Recently we had argued that Cyg X-3 is an archetypical black hole binary and have compared its X-ray/ radio emission properties with the other known black hole binaries (Choudhury et al. 2003) and hence it is instructive to compare the PDS of Cyg X-3 at very low frequencies. 


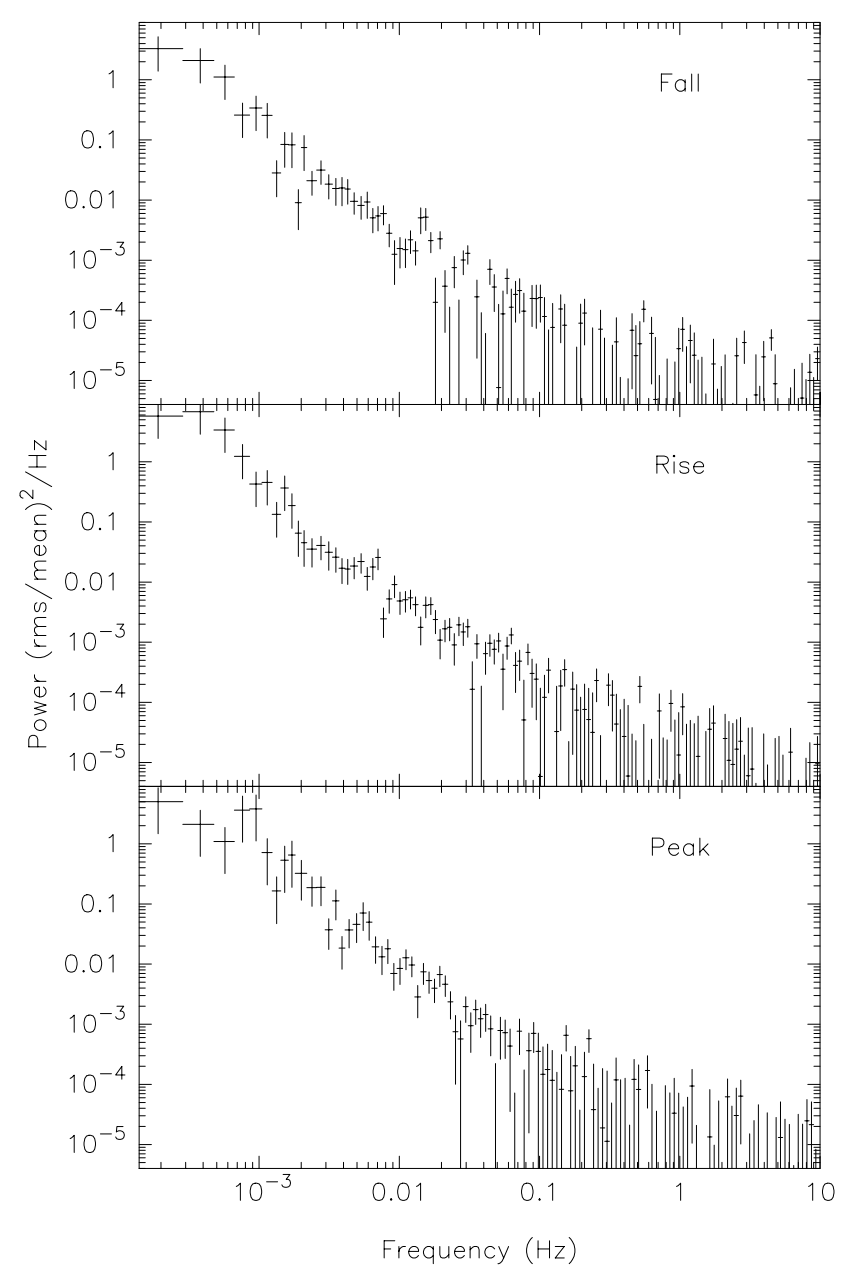

Fig. 4. Power Density Spectrum (PDS) of Cygnus X-3 for the RXTE-PCA pointed observations shown in Fig. 3, after correcting for the binary variations. The PDS is generated separately for three regions of the binary phase namely the fall (phase 0.75-1.0 - top panel), rise (phase 0-0.4 - middle panel) and the peak (phase 0.4-0.75bottom panel).

We have derived the PDS of Cyg X-3 at very low frequencies using RXTE-ASM data using a method similar to that followed by Reig et al.(2002). The results are given in Fig. 5, along with the PDS obtained using the pointed observations of RXTE-PCA. It is found that below $5 \times 10^{-7} \mathrm{~Hz}$, the power-law index is flatter $(0.97 \pm 0.17)$, consistent with that obtained for Cyg X-1 and GRS 1915+105. The remarkable feature of Cyg X-3, however, is the low power - about 3.5\% in this frequency range. The flatter power law at low frequencies $\left(<10^{-6} \mathrm{~Hz}\right)$ and the steeper power-law at higher frequencies $\left(\left(>10^{-3} \mathrm{~Hz}\right)\right.$ intercept at $\sim 10^{-4} \mathrm{~Hz}$ signifying a break in the power spectrum. Though there is a hump like feature at this frequency, we cannot rule out the influence of the binary period $\left(\sim 10^{-4} \mathrm{~Hz}\right)$ on the shape of this hump. A long uninterrupted observation would be required to address this issue.

\section{Radio X-ray correlation in Cygnus X-3}

To avoid the possible effects of binary modulation, the analysis for deriving the interesting correlation between the X-ray and

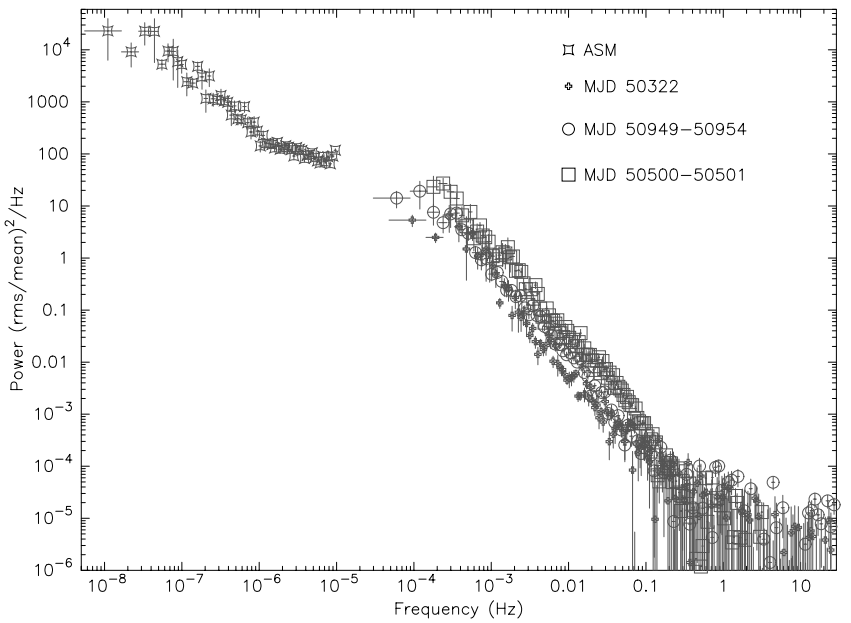

Fig. 5. The PDS of Cygnus X-3 over a wide frequency range obtained using RXTE-ASM and RXTE-PCA at three observation periods.

radio emission in Cygnus X-3, as reported by Choudhury et al. (2002), was done using data averaged over 10 days. Here we repeat the same analysis for the binary corrected X-ray emission. The Spearman partial rank correlation test is used to determine the correlation between two or more variables. The partial rank coefficient is computed from the sampling distribution which may be derived by analogy with a parametric statistic (Macklin 1982). For correlation among three variables, say A, $\mathrm{X}$ and $\mathrm{Y}$, the null-hypothesis is that the correlation between $\mathrm{A}$ and $\mathrm{X}$ arises entirely from those of $\mathrm{Y}$ with $\mathrm{A}$ and $\mathrm{X}$ separately. The value of the correlation coefficient lies between -1 and 1 . The negative value signifies anti-correlation. The significance level associated with the correlation between $\mathrm{A}$ and $\mathrm{X}$, independent of $\mathrm{Y}$, is given by the $D$-parameter, which is normally distributed about zero with unit variance if the null-hypothesis, that the $\mathrm{A}-\mathrm{X}$ relation arises entirely from those of $\mathrm{Y}$ with $\mathrm{A}$ and $\mathrm{X}$ separately, is true. The correlation coefficient and other parameters (see Choudhury et al. 2002, 2003) are given in Table 2 for integration time of data points ranging from 1 to 15 days. The null-hypothesis probability, as a function of bin size, is plotted in Fig. 6 where it appears that the correlation becomes stronger for smaller bin sizes due to increase in the number of degrees of freedom. Hence, evidently, both from Table 2 and Fig. 6, the correlation time scale is shorter than one day, which is not surprising because for a binary period of $4.8 \mathrm{~h}$ the possible time scales which can come into play, viz. the viscous time scale of the accretion disc, the variability time scale of the accretion disc corona, and the time scale for variation of the jet emission must be smaller than a day.

\section{Discussion}

\subsection{The binary template and its stability}

A noteworthy feature of Cygnus X-3 is that the binary period has remained consistent for more than 25 years with a linear decay with no second derivative of the period (Singh et al. 2002). The relative phasing of the X-ray and infra-red emissions from this source, coupled by the orbital evolution of the 


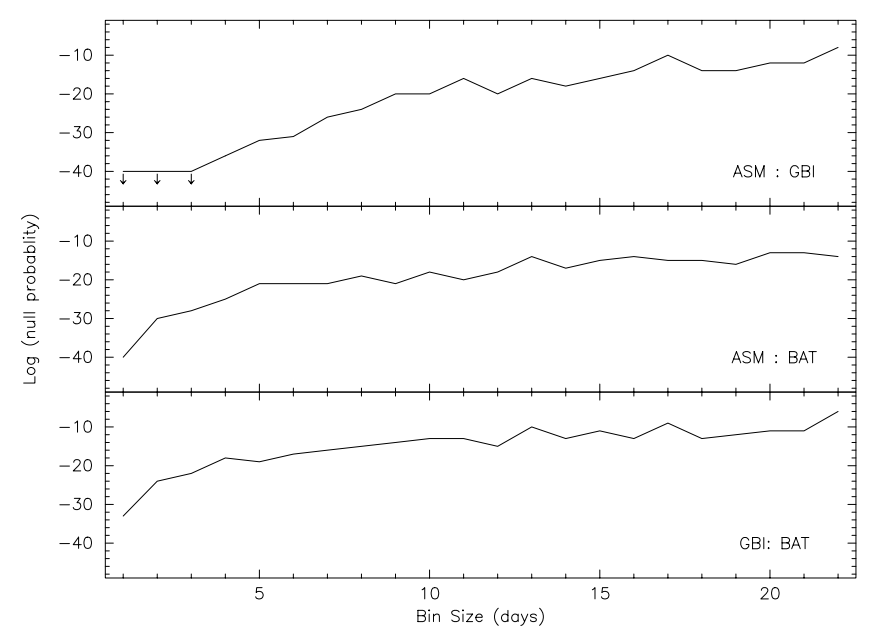

Fig. 6. The null-hypothesis probability for not having a correlation between the soft X-ray (ASM), radio (GBI) and hard X-ray (BATSE) emissions in Cygnus X-3 for various bin sizes of integration. The first three points in the top panel denote the upper limits.

line shift of the He I emission lines, gives the picture that the binary modulation is due to the orbital motion of the ionized two temperature wind originating in the companion Wolf-Rayet star irradiated by the X-rays from the compact object (van Kerkwijk 1993). The minimum occurs when the cooler part of the wind in the shadow of the Wolf-Rayet companion is in the line of sight of the observer with the compact object at the superior conjunction. The asymmetry in the binary modulation profile may be due to 1) eccentricity in the binary orbit, and/or 2) asymmetric distribution of matter within the system (Elsner et al. 1980). Given the fact that both the binary period and the template have remained consistent for a period of $\sim 25$ years, the presence of any reasonable apsidal motion (Ghosh et al. 1981) may be ruled out (van der Klis \& Bonnet-Bidaud 1989). Hence, eccentricity, if present in the binary orbit, is negligible to effect any asymmetry in the lightcurve. Therefore it is more likely that an asymmetric distribution of matter, causing an asymmetric distribution of optical depth, introduces the asymmetry in the binary template. In this scenario the orbital period decay is explained by the loss of angular momentum via the wind from the Wolf-Rayet companion.

Since the early days of its observation, it is believed that in this source the X-ray emission is extensively reprocessed, either in the stellar wind (Davidsen \& Ostriker 1974; Hertz et al. 1978; Becker et al. 1978) or in a cocoon (X-ray halo) surrounding and extending beyond the binary system (Milgrom 1976; Predehl et al. 2000). There also exist models of X-ray reprocessing in the accretion disk corona (White \& Holt 1982; Molnar \& Mauche 1986) where the low energy photons are upscattered by Comptonization also producing fluorescence of ionized Fe (Rajeev et al. 1994). Nakamura et al. (1993) explain the X-ray spectra as obtained by the Ginga observatory by proposing the presence of three species of ionized gas, fully ionized, almost fully ionized and nearly ionized, engulfing the binary system. Fender et al. (1999) suggest a WR type wind with the geometry of a disc in the binary plane with a size much bigger than the binary system to be the origin of the binary modulated He emission lines (obtained from infra-red spectra). This wind originates from the companion which is a WN type Wolf-Rayet star. Polarimetric study of the $K$-band lightcurve also suggests a preferential plane of scattering. In this model the X-ray emission undergoes scattering in this disc-like two temperature wind (van Kerkwijk 1993), resulting in the asymmetry in the binary template.

The residue of the (binary) folded lightcurve (Fig. 2) may be attributed to the long term variation of the X-ray emission by virtue of the change in wind and/or cocoon mass distribution, including various state changes from soft (and high) to low (and hard) and vice-versa, accompanied by the correlated radio flares. One small aspect not considered so far is the generally random fluctuation in the X-ray lightcurve at the peak of the binary ephemeris. A very detailed analysis of the nature of this fluctuation may help in determining the geometrical and physical structure of the accreting system involving the wind from the companion and the X-ray halo (Predehl et al. 2000) engulfing the system. Recently, Stark \& Saia (2003) have attempted to constrain the binary orbital parameters by measuring the line shift of Helium $\alpha$ line of Fe XXV and Lyman $\alpha$ lines of Si XIV and S XVI as a function of the orbital phase. By ascribing the Fe line to originate very close to the compact object surface and the $\mathrm{Si}$ and $\mathrm{S}$ line to originate from the wind from the companion, they constrain the masses of the companion and the compact object to be $\leq 7.3 M_{\odot}$ and $\leq 3.6 M_{\odot}$, respectively, provided the orbital inclination is small $\left(i=24^{\circ}\right.$ - this agrees with the limit $0-40^{\circ}$ of Fender et al. 1999). The phase resolved spectra of the various Fe emission lines (6.4 keV, $6.7 \mathrm{keV}$ and $6.9 \mathrm{keV})$ and the absorption edges (7.1 keV and $9.1 \mathrm{keV})$ during both low-hard and high-soft states may provide better constraints on the origin of the Fe emission lines. The binary modulation of the emission lines reported by Stark \& Saia (2003) need to be fit properly with the binary template (Singh et al. 2002) to obtain a more definitive mass function of the system. A long continuous X-ray observation of the source spanning a few orbital periods with very high resolution X-ray spectra will provide a better binary profile which is essential for constraining the binary parameters and obtaining the geometrical and physical structure of the system.

\subsection{Power Density Spectrum}

The distinct feature of the power density spectra of the source is the shifting of the spectra towards low frequency regime (vis-a-vis the "normal" frequency regime of other Galactic $\mathrm{X}$-ray binaries), corresponding to very massive black hole systems (Hayashida et al. 1998; Czerny et al. 2001). Sunyaev \& Revnivtsev (2000) have compiled the power density spectra (multiplied by frequency) of the most common Galactic X-ray binaries, both neutron stars and black hole candidates (in their low/hard states). The black hole binaries show, typically, a power law dependence with a positive index in the region of $\sim 0.01-1 \mathrm{~Hz}$, flat spectra for the next decade of frequency range, followed by a power law decay (i.e. negative index) of power in the $\sim 10-100 \mathrm{~Hz}$. The PDS of the neutron stars is generally shifted towards the higher frequency region by an 
Table 1. The Spearman Rank Correlation coefficient, the null-hypothesis probability for no correlation and the $D$-parameter for the effect of the third parameter, among the radio (GBI), soft X-ray (ASM) and hard X-ray (BAT) emission of Cygnus X-3, in the low-hard state with the observed flux averaged for various bin sizes.

\begin{tabular}{ccccccccccc}
\hline \hline $\begin{array}{c}\text { Bin size } \\
\text { (days) }\end{array}$ & No. of & \multicolumn{3}{c}{ Spearman Rank Corr. Coeff. } & \multicolumn{3}{c}{ Null-hypothesis probability } & \multicolumn{3}{c}{$D$ parameter } \\
\hline 1 & 638 & 0.70 & -0.50 & -0.45 & $<.10 \mathrm{E}-40$ & $.28 \mathrm{E}-40$ & $.68 \mathrm{E}-33$ & 17.94 & -7.31 & -4.37 \\
2 & 356 & 0.73 & -0.57 & -0.51 & $<.10 \mathrm{E}-40$ & $.15 \mathrm{E}-30$ & $.49 \mathrm{E}-24$ & 13.83 & -6.38 & -3.22 \\
3 & 246 & 0.76 & -0.64 & -0.58 & $<.10 \mathrm{E}-40$ & $.13 \mathrm{E}-28$ & $.12 \mathrm{E}-22$ & 11.15 & -6.11 & -3.08 \\
4 & 186 & 0.77 & -0.68 & -0.60 & $.36 \mathrm{E}-36$ & $.12 \mathrm{E}-25$ & $.27 \mathrm{E}-18$ & 9.62 & -6.26 & -2.17 \\
5 & 149 & 0.79 & -0.69 & -0.66 & $.28 \mathrm{E}-32$ & $.23 \mathrm{E}-21$ & $.79 \mathrm{E}-19$ & 8.73 & -4.64 & -3.11 \\
6 & 125 & 0.82 & -0.73 & -0.68 & $.49 \mathrm{E}-31$ & $.41 \mathrm{E}-21$ & $.20 \mathrm{E}-17$ & 8.54 & -4.75 & -2.32 \\
7 & 108 & 0.82 & -0.77 & -0.70 & $.50 \mathrm{E}-26$ & $.19 \mathrm{E}-21$ & $.25 \mathrm{E}-16$ & 7.18 & -5.32 & -2.06 \\
8 & 94 & 0.83 & -0.77 & -0.72 & $.63 \mathrm{E}-24$ & $.62 \mathrm{E}-19$ & $.17 \mathrm{E}-15$ & 6.80 & -4.60 & -2.25 \\
9 & 84 & 0.81 & -0.83 & -0.74 & $.95 \mathrm{E}-20$ & $.16 \mathrm{E}-21$ & $.15 \mathrm{E}-14$ & 5.27 & -6.05 & -1.74 \\
10 & 75 & 0.84 & -0.82 & -0.73 & $.26 \mathrm{E}-20$ & $.56 \mathrm{E}-18$ & $.99 \mathrm{E}-13$ & 6.17 & -5.12 & -1.19 \\
11 & 69 & 0.81 & -0.86 & -0.75 & $.20 \mathrm{E}-16$ & $.19 \mathrm{E}-20$ & $.76 \mathrm{E}-13$ & 4.33 & -6.26 & -1.46 \\
12 & 63 & 0.87 & -0.86 & -0.82 & $.88 \mathrm{E}-20$ & $.33 \mathrm{E}-18$ & $.19 \mathrm{E}-15$ & 5.09 & -4.25 & -2.26 \\
13 & 58 & 0.85 & -0.81 & -0.74 & $.24 \mathrm{E}-16$ & $.74 \mathrm{E}-14$ & $.31 \mathrm{E}-10$ & 5.54 & -4.26 & -1.13 \\
14 & 54 & 0.89 & -0.87 & -0.82 & $.63 \mathrm{E}-18$ & $.70 \mathrm{E}-17$ & $.19 \mathrm{E}-13$ & 4.90 & -4.33 & -1.61 \\
\hline
\end{tabular}

order of magnitude. From the PDS of Cygnus X-3 (Fig. 4) it is quite apparent that, from the RXTE-PCA pointing observations, we are catching the power law decay portion of the PDS, shifted by a few decades in the lower frequency regime, with the power getting merged with the white noise at $\sim 0.1 \mathrm{~Hz}$. Due to improved continuous observational capabilities of RXTE it is possible to extend the PDS to the lower frequency regime $\left(\sim 10^{-4} \mathrm{~Hz}\right)$ and hence ascertain from the wide frequency band PDS that, in general, the power above $\sim 0.1 \mathrm{~Hz}$ is not discernible from the white noise. One may reconcile the absence of power in the high frequency regime to the scattering of the X-ray photons in the wind from the companion WolfRayet star, reducing the amplitude of the fast X-ray variability (Berger \& van der Klis 1994). If we reconcile the absence of power above $0.1 \mathrm{~Hz}$ to the reprocessing of the X-ray emission in the circumstellar environment, then this feature does provide an interesting sidelight to the paradigm which states that the variability time-scale scales linearly with the mass of the compact object (Hayashida et al. 1998; Czerny et al. 2001), and introduce an additional factor, viz. reprocessing in the dense circumstellar material, where it exists, into the picture. It is also interesting that the PDS at very low frequencies have about an order of magnitude lower power compared to other black hole candidate sources Cyg X-1 and GRS 1915+105.

\section{3. $X$-ray radio correlations}

Correlation between soft X-ray and radio emission has been noted in many black hole binary sources (Gallo et al. 2002) and finding similar correlation in Cygnus X-3 (Choudhury et al. 2002) firmly puts Cygnus $X-3$ as a good candidate for harboring a black hole. Choudhury et al. (2003) point out that correlation between the X-ray and radio emission and the anti-correlation between the soft and hard X-rays are directly related to a causal connection between the spectral shape in the $\mathrm{X}$-rays and the radio emission. They have shown that such a picture consistently explains the observed behaviors of three bright Galactic X-ray binary sources Cygnus X-3, Cygnus X-1 and GRS $1915+105$. By correcting the X-ray light curve for the binary variations, we have shown that the correlation time scale is shorter than a day (Table 1 and Fig. 6). Therefore it is necessary to analyze the pointed continuous observation in the X-ray wide-band in order to determine the dynamic time scale of soft and hard X-ray emission. This will enable us to understand the detailed structure of the accretion disk, and is the focus of our future work. A simultaneous radio observation will be imperative in understanding the detailed mechanism of the disk jet connection in the system, which will provide a quantitative estimate of the extent of jet power being emitted in the X-ray band.

\section{Conclusions}

In this paper we have suggested a method to correct the X-ray light curve of Cygnus X-3 for the binary modulation. The binary period shows consistency over a long period of 25 years, with minor variation in the asymmetric binary phase profile. An X-ray halo consisting of photoionized plasma originating from the wind of the companion Wolf-Rayet type companion star reprocesses the X-ray emission, while any eccentricity of the binary orbit which may cause asymmetry in the X-ray binary profile is minimal because of absence of any detectable apsidal motion over the long period of $\sim 25$ years.

We have also examined the binary corrected light curve for short term variabilities and noted that the shape of the PDS follows the power law behavior in both low and high states, 
similar to other Galactic black hole sources. Most significantly there exists no power at frequency $>0.1 \mathrm{~Hz}$, with the PDS being shifted to the low frequency regime vis-a-vis the Galactic X-ray binaries, both black hole and neutron stars. This is a unique feature of this enigmatic source.

Using the corrected light curve we have examined the X-ray radio correlations at various time scales and have concluded that the time scale is shorter than a day. The soft and hard $\mathrm{X}$-ray correlation need to be examined using the binary corrected RXTE pointed (PCA and HEXTE) data covering a time scale of a few hours or longer to unambiguously determine the time scale of anti-correlation, and hence determine the detailed physical as well geometrical structure of the disk.

Acknowledgements. This research has made use of data obtained through the HEASARC Online Service, provided by the NASA/GSFC, and the Green Bank Interferometer, a facility of the National Science Foundation operated by the NRAO in support of NASA High Energy Astrophysics Programs. M.C. and S.V.V. have been partially supported by the Kanwal Rekhi Scholarship for Career Development. AKJ is grateful to P. S. Goel, Director, ISAC and K. Kasturirangan, Chairman, ISRO, for their constant encouragement and support during the course of this work. The authors acknowledge the editor A. Jones for his continued patient attention, and the referee M. Stark for very detailed assessment and extremely critical revision of the paper.

\section{References}

Becker, R. H., Robinson-Saba, J. L., Boldt, E. A., et al. 1978, ApJ, 224, L113

Becklin, E. E., Neugebauer, G., Hawkins, F. G., et al. 1973, Nature, 245,302

Berger, M., \& van der Klis, M. 1994, A\&A, 292, 175

Bonnet-Bidaud, J. M., \& Chardin, G. 1988, Phys. Rep., 170, 325

Choudhury, M., Rao, A. R., Vadawale, S. V., Ishwara-Chandra, C. H., \& Jain, A. K. 2002, A\&A, 383, L35

Choudhury, M., Rao, A. R., Vadawale, S. V., \& Jain, A. K. 2003, ApJ, 593,452

Czerny, B., Nikolajuk, M., Piasecki, M., et al. 2001, MNRAS, 325, 865

Davidsen, A., \& Ostriker, J. P. 1974, ApJ, 189, 331

Elsner, R. F., Ghosh, P., Darbro, W., et al. 1980, ApJ, 239, 335
Fender, R. P., Bell Burnell, S. J., Waltman, E. B., et al. 1997, MNRAS, 288, 849

Fender, R. P., Hanson, M. M., \& Pooley, G. 1999, MNRAS, 308, 473

Gallo, E., Fender, R. P., \& Pooley, G. G. 2002, Proc. of the 4th Microquasar Workshop, ed. Ph. Durouchoux, Y. Fuchs, \& J. Rodriguez (Center for Space Physics: Kolkata), 201

Ghosh, P., Elsner, R. F., Weisskopf, M. C., et al. 1981, ApJ, 251, 230

Giacconi, R., Gorenstein, P., Gursky, H., \& Waters, J. R. 1967, ApJ, 148, L119

Gregory, P. C., Kronberg, P. P., Seaquist, E. R., et al. 1972, Nature, 239,440

Hayashida, K., Miyamoto, S., Kitamoto, S., et al. 1998, ApJ, 500, 642

Hertz, P., Joss, P. C., \& Rappaport, S. 1978, ApJ, 224, 614

Kitamoto, S., Miyamoto, S., \& Matsui, W. 1987, PASJ, 39, 259

Kitamoto, S., Kawashima, K., Negoro, H., et al. 1994, PASJ, 46,105

Macklin, J. T. 1982, MNRAS, 199, 1119

McCollough, M. L., Robinson, C. R., Zhang, S. N., et al. 1999, ApJ, 517,951

Milgrom, M. 1976, A\&A, 51, 215

Mitra, A. 1996, MNRAS, 280, 953

Molnar, L. A., \& Mauche, C. W. 1986, ApJ, 310, 343

Nakamura, H., Matsuoka, M., Kawai, N., et al. 1993, MNRAS, 261, 353

Paerels, F., Cottam, J., Sako, M., et al. 2000, ApJ, 533, L135

Parsignault, D. R., Gursky, H., Kellog, E. M., et al. 1972, Nat. Phys. Sci., 239, 123

Predehl, P., Burwitz, V., Paerels, F., et al. 2000, A\&A, 357, L25

Rajeev, M. R., Chitnis, V. R., Rao, A. R., et al. 1994, ApJ, 424, 376

Rao, A. R., Agrawal, P. C., \& Manchanda, R. K. 1991, A\&A, 241, 127

Reig, P., Papadakis, I., \& Kylafis, N. D. 2002, A\&A, 383, 202

Schmutz, W., Geballe, T. R., \& Schild, H. 1996, A\&A, 311, L25

Singh, N. S., Naik, S., Paul, B., et al. 2002, A\&A, 392,161

Stark, M. J., \& Saia, M. 2003, ApJ, 587, L101

Sunyaev, R. A., \& Revnivtsev, M. 2000, A\&A, 358, 617

van der Klis, M., \& Bonnet-Bidaud, J. M. 1981, A\&A, 95, L5

van der Klis, M., \& Bonnet-Bidaud, J. M. 1982, A\&AS, 50, 129

van der Klis, M., \& Jansen, F. A. 1985, Nature, 313, 768

van der Klis, M., \& Bonnet-Bidaud, J. M. 1989, A\&A, 214, 203

van Kerkwijk, M. H., Charles, P. A., Geballe, T. R., et al. 1992, Nature, 355, 703

van Kerkwijk, M. H. 1993, A\&A, 276, L9

Waltman, E. B., Ghigo, F. D., Johnston, K. J., et al. 1995, AJ, 110, 290

Watanabe, H., Kitamoto, S., Miyamoto, S., et al. 1994, ApJ, 433, 350

White, N. E., \& Holt, S. S. 1982, ApJ, 257, 318 